\title{
Association between incidence of atrial fibrillation and duration of cardiopulmonary bypass in coronary artery bypass graft surgery (CABG): a cohort study
}

\author{
Maryam Rajabi ${ }^{1}$, Gholamreza Safarpoor ${ }^{2}$, Seyed Reza Borzou ${ }^{3}$, Maryam Farhadian ${ }^{4}$, Arezo Arabi ${ }^{5}$, Aliasghar \\ Moeinipour ${ }^{6}$, Babak Manafi $^{7}$
}

\begin{abstract}
${ }^{1}$ M.Sc. in Nursing, Nursing and Midwifery School, Hamedan University of Medical Sciences, Hamedan, Iran
${ }^{2}$ Assistant Professor, Department of Cardiac Surgery, Faculty of Medical Sciences, Farshchian Heart Center, Hamedan University of Medical Sciences, Hamedan, Iran

${ }^{3}$ Assistant Professor, Department of Medical Surgical Nursing, Member of Chronic Disease (Home Care) Research Center, School of Nursing and Midwifery, Hamedan University of Medical Sciences, Hamedan, Iran

${ }^{4}$ Assistant Professor of Biostatistics, Modeling of Non Communicable Diseases Research Center, Department of Biostatistics, School of Public Health, Hamedan University of Medical Sciences, Hamedan, Iran

${ }^{5}$ Master of Epidemiology, Hamedan University of Medical Sciences, Hamedan, Iran

${ }^{6}$ Assistant Professor of Cardiovascular Surgery, Faculty of Medicine, Mashhad University of Medical Sciences, Mashhad, Iran

${ }^{7}$ Assistant Professor of Cardiovascular Surgery, Faculty of Medicine, Hamedan University of Medical Sciences. Iran
\end{abstract}

Type of article: Original

\begin{abstract}
Background: Open heart surgery is one of the most common and valuable treatment methods for cardiovascular diseases, a common side effect of which is atrial fibrillation that occurs due to various reasons.

Objective: To determine the relationship between incidence of atrial fibrillation (AF) and duration of cardiopulmonary bypass (CPB) in patients after open heart surgery.

Methods: The present retrospective cohort study was conducted on 330 patients in Farshchian Heart Center through census. The required data were collected from medical records of the patients undergoing coronary bypass surgery using data collection between April 2015 and March 2015. Then, data analysis was performed using SPSS software (ver.16) at error level of $p<0.05$. The tests used in this study included independent-samples t-test, Mann-Whitney, and chi-squared tests.

Results: Based on the results, mean age of the patients was $61.76 \pm 9.2$, the majority of the patients $(70.1 \%)$ were male. The association between Incidence of Atrial Fibrillation and cardiopulmonary pump time (minute) was not meaningful. Incidence of atrial fibrillation had statistically significant relationship with variables of mean age, BMI, PAC, PVC, creatinine and duration of hospitalization $(\mathrm{p}<0.05)$; on the other hand, variables of gender, cross clamp time (minute), intubation time (hour), and clinical history had no effect on atrial fibrillation incidence rate $(\mathrm{p}>0.05)$.

Conclusion: Since the pathogenesis of AF after cardiac surgery is believed to be multifactorial, including clinical variables and technical intraoperative factors, the relation between incidence of AF with mean age, BMI, PAC, PVC, creatinine and duration of hospitalization was significant. But AF was not related to cardiopulmonary pump time (minute). It is necessary to conduct further research on factors affecting incidence of atrial fibrillation.
\end{abstract}

Keywords: Cardiopulmonary bypass, Atrial fibrillation, Heart surgery, Cardiovascular diseases

\section{Corresponding author:}

Assistant Professor Dr. Babak Manafi, Faculty of Medicine, Hamedan University of Medical Sciences, Hamedan. Iran. Postal code: 9137913316, Tel/Fax: +98.51138525307, h/p: +989151108457, Email: Manafi@umsha.ac.ir

Received: April 19, 2017, Accepted: December 22, 2017, Published: April 2018

iThenticate screening: December 24, 2017, English editing: March 01, 2018, Quality control: XXX

This article has been reviewed / commented by three experts

(C) 2018 The Authors. This is an open access article under the terms of the Creative Commons Attribution-NonCommercialNoDerivs License, which permits use and distribution in any medium, provided the original work is properly cited, the use is non-commercial and no modifications or adaptations are made. 


\section{Introduction}

Cardiovascular diseases (CVDs) are the main cause of mortality and disability worldwide (1, 2). IHD (ischemic heart disease) is a common CVD and one of the main causes of mortality in the world that, with a growing increase rate, has become a major health problem. According to the reports of the WHO, this disease accounts for 1.7 million deaths per year, which is estimated to reach 11.1 million by 2020 (3). Besides being a common cause of mortality in developed countries, IHD, next to accidents, is the major cause of deaths in Iran (4). There are various treatments for heart diseases, one of the most common and valuable of which is CABG (coronary arteries bypass grafting), in addition to medical treatments (5). CABG is one of the most recent proposed treatments for patients with coronary arteries constriction (6), which can play a fundamental role in reducing the mortality rate and complications caused by the disease, if performed at the appropriate time (4). CABG may be performed as an open or closed heart surgery (using cardiopulmonary bypass [CPB] pump or without it) (7). Regardless of the increasing numbers of "off-pump" CABG and interventional therapy, CPB technique is still an essential assisting method for open heart surgery (8). Similar to other invasive treatments and surgeries, CAGB may also cause some side effects. Due to its difficult nature or probable heart stoppage and establishment of extracorporeal blood circulation, despite being effective and efficient, CAGB would lead to certain side effects potentially during the operation and after it (7). In this type of surgery, the heart blood flow is blocked and its flow is controlled by CPB machine at a rate of $2-4 \mathrm{~L} / \mathrm{min}$ at mild hypothermia $\left(34{ }^{\circ} \mathrm{C}\right)$ for coronary artery bypass grafting $\left.(\mathrm{CABG})\right)$ and hematocrit at $18 \%$ to $25 \%$. Myocardial protection was achieved by the administration of cold, multidose blood cardioplegia infused through the aortic root and the coronary sinus (9-11). Several studies have shown that "off-pump" (CABG) might be associated with better early clinical outcomes compared with use of $\mathrm{CPB}$, including reduction in the occurrence of pulmonary complications, reoperations for bleeding, transfusion requirements and atrial fibrillation (9). It is in fact, well known that surgical stress in use of CPB can cause various side effects including systemic inflammatory response, nervous side effects, cardiac ischemia, renal failure, hemodynamic instability, and lung dysfunction (9, 12, 13). Several associated factors including hypothermia, hemodilution, electrolyte imbalance, and pharmacological agents used during surgery with $\mathrm{CPB}$, cause these complications (14). In CABG with CPB, hemodilution causes more stress which leads to increased levels of plasma cortisol within 24 hours after the surgery (15). Open-heart surgery with the use of cardiopulmonary bypass (CPB) is associated with significant inflammation compared to off-pump CABG (14, 15). CPB provokes systemic and non-systemic inflammatory responses, which increases inflammatory cytokines, endotoxin and metabolic products (12, 14-16). These changes can cause postoperative adverse effects and complications, such as delirium, cognitive dysfunction, impaired immune system and increased oxygen consumption, catabolism, ischemic reperfusion injury, oxidative stress, and neurohormonal activation and length of hospital stay $(12,15)$. However, myocardial ischemic, cardioplegic arrest and reperfusion has been implicated as a major trigger of tissue damage and inflammatory response (14). One of the most common complications after CABG, is Atrial Fibrillation (AF) (17-19). The incidence of postoperative AF varies from 10-50\% (17, 18, 20, 21). It usually tends to occur within 2 to 4 days after the operation $(17,20)$. Numerous studies, mainly retrospective, have been conducted to clarify the pathogenesis of postoperative AF as well as to identify predisposing factors. However, the exact etiologic pattern still remains unclear (22). The proposed contributory factors include inflammation triggered by cardiopulmonary bypass, beta-blocker withdrawal, hyperthyroidism, caffeine, right coronary artery stenosis, atrial ischemia, inadequate intraoperative cardiac protection, perioperative ischemic injury, postoperative pericarditis, autonomic imbalance, and fluid/electrolyte disturbances during the intra-and post operative periods (22, 23). It is assumed that one of the reasons of POAF may be the post-operative local and systemic inflammations (24). Although POAF is typically self-limiting $(17,25,26)$, its adverse complications including hemodynamic disorders, cardiac failure, thromboembolism, increased postoperative stroke, nervous and renal complications, the use of inotropes, and increases in duration of hospitalization are important $(16,22,26-28)$. Such complications are a potential for prolongation of hospitalization in hospitals and special units as well as increased costs (20, 22, 24, 29). Prescribing anti-arrhythmic drugs, preventing the disease, and recognizing its effective factors can help in reducing treatment costs, duration of hospitalization, as well as other complications of POAF (24). Thus, it is probable that the CPB (cardiopulmonary bypass) response might help creation of postoperative AF. As it was stated, there are conflicting evidences on the effect of bypass time on AF; therefore, this subject can be investigated by conducting a specific study in this regard. Based on the above-mentioned reasons, finding an efficient method to prevent this disease can effectively help in reducing the duration of hospitalization, treatment costs, as well as incidence rate of this disease. Accordingly, the present study was aimed to determine the relationship between incidence of AF and duration of CPB pump in patients after open heart surgery. 


\section{Material and Methods}

The present retrospective cohort study was conducted in Farshchian Heart Center, Hamedan University of Medical Sciences. Research population included all the patients undergoing open heart surgery between April 2015 and March 2015. Data collection was performed through census; furthermore, the research was conducted on all the patients who had the following inclusion and exclusion criteria. The inclusion criteria included patients who underwent open heart surgery, availability of medical records, and completeness of the required information; The exclusion criteria included simultaneous mitral valve surgery, history of heart surgery, preoperative supraventricular dysrhythmia, consumption of anti-arrhythmic drugs (except for beta-blockers) during, before, and after surgery, having serious lung problems (patients who received lung counseling by request of the physician and were high-risk in terms of lung diseases), chronic renal failure, history of ventricular aneurysm surgery, thyroid dysfunction, age of above 80 years old, and history of OFF-Pump surgery. Finally, after examining all the cases, 330 cases were selected and 69 were excluded due to having the exclusion criteria (Diagram-1). Data collection tools included demographic information and clinical information. Demographic information included age, gender, weight, height, and body mass index (BMI); furthermore, clinical information included NA, K, creatinine, incidence of atrial fibrillation (AF) and other arrhythmias, cardiac infarction, systemic diseases, duration of cardiopulmonary bypass (CPB), duration of cross clamp and intubation length of hospital stay. According to the questionnaires, a specific code was allocated to each patient. It must be noted that all the required information was collected from cases of the hospitalized patients; additionally, all the ethical considerations were applied throughout the study. Regarding the retrospectiveness of the study, it was impossible to obtain the patients' informed consent. Information of the patients' cases was considered confidential and merely used for the research objectives. At the end, the collected data was analyzed using independent-samples t-test, Mann-Whitney, and chi-squared tests; furthermore, analysis of the obtained data was performed using SPSS version 16 (SPSS Inc., Chicago, Illinois, USA) at error level of p $<0.05$.

\section{Results}

Based on the results related to the demographic information, the mean age of the studied patients was $61.76 \pm 9.2$; moreover, the majority of the patients $(70.1 \%)$ were male. Also, $72 \%$ of them had three involved arteries. Incidence of AF was observed in $32.6 \%$ of the patients in ICU.

Table 1. Demographic and clinical characteristics of patients

\begin{tabular}{|c|c|c|c|c|}
\hline Variables & AF incidence & $\mathrm{n}$ & Mean \pm SD & $\begin{array}{l}\text { Independent-samples t- } \\
\text { test p-value }\end{array}$ \\
\hline \multirow[t]{2}{*}{ Age (year) } & Yes & 86 & $63.95 \pm 9.9$ & \multirow[t]{2}{*}{0.008} \\
\hline & No & 176 & $60.70 \pm 8.7$ & \\
\hline \multirow[t]{2}{*}{ BMI kg/m² } & Yes & 83 & $23.33 \pm 3$ & \multirow[t]{2}{*}{0.001} \\
\hline & No & 171 & $25.70 \pm 4.9$ & \\
\hline \multirow[t]{2}{*}{ Male* } & Yes & 66 & - & \multirow[t]{2}{*}{0.065} \\
\hline & No & 117 & - & \\
\hline \multirow[t]{2}{*}{ Sodium } & Yes & 85 & $5.1 \pm 140.89$ & \multirow[t]{2}{*}{0.595} \\
\hline & No & 176 & $140.60 \pm 3.6$ & \\
\hline \multirow[t]{2}{*}{ Potassium } & Yes & 85 & $4.1 \pm 0.56$ & \multirow[t]{2}{*}{0.791} \\
\hline & No & 176 & $4 \pm 0.46$ & \\
\hline \multirow[t]{2}{*}{ Creatinine } & Yes & 85 & $1.2 \pm 0.36$ & \multirow[t]{2}{*}{0.0001} \\
\hline & No & 176 & $1.03 \pm 0.22$ & \\
\hline \multirow[t]{2}{*}{ Ejection fraction } & Yes & 85 & $44.8 \pm 8$ & \multirow[t]{2}{*}{0.257} \\
\hline & No & 167 & $45.9 \pm 6.4$ & \\
\hline \multirow{2}{*}{$\begin{array}{l}\text { Duration of cardiac bypass pump } \\
\text { (minute) }\end{array}$} & Yes & 84 & $56.73 \pm 19.13$ & \multirow[t]{2}{*}{0.746} \\
\hline & No & 170 & $55.90 \pm 19.27$ & \\
\hline \multirow[t]{2}{*}{ Duration of cross clamp (minute) } & Yes & 84 & $34.11 \pm 12.5$ & \multirow[t]{2}{*}{0.502} \\
\hline & No & 170 & $32.95 \pm 13.20$ & \\
\hline \multirow[t]{2}{*}{ Duration of intubation (hour) } & Yes & 84 & $9.30 \pm 2.76$ & \multirow[t]{2}{*}{0.261} \\
\hline & No & 170 & $9.63 \pm 1.87$ & \\
\hline \multirow[t]{2}{*}{ Length of hospital stay** } & Yes & 74 & $6.12 \pm 1.57$ & \multirow[t]{2}{*}{0.013} \\
\hline & No & 170 & $5.67 \pm 1.12$ & \\
\hline
\end{tabular}

*Chi-square test was used in this variable; **Mann-Whitney test was used in this variable 
Moreover, data analysis showed that the mean age of the patients in the AF incidence group was higher than that of the patients without incidence of $\mathrm{AF}(\mathrm{p}=0.008)$; furthermore, BMI of the patients in the AF incidence group was significantly lower than that in the other group $(\mathrm{p}<0.001)$. However, variable of gender had no effect on incidence of AF ( $>0.05$ ). Comparing the clinical results indicated that the mean value of creatinine in patients of the AF incidence group was significantly higher than that of the patients without AF ( $\mathrm{p}=0.0001)$; on the other hand, the mean duration of hospitalization (in days) for patients with AF was significantly higher than that of the patients without $\mathrm{AF}(\mathrm{p}=0.013)$. But variables such as ejection fraction, $\mathrm{Na}, \mathrm{K}$, duration of cross clamp (min), duration of cardiac bypass pump (min) and duration of intubation (h) had no effect on incidence of AF ( $>0.05)$ (Table 1). Results obtained from the patients' information showed that the PAC $(p<0.001)$ and PVC $(p=0.010)$ arrhythmias were significantly related with incidence of AF; however, supraventricular tachycardia had no effect on incidence of AF $(\mathrm{p}=0.548)$ (Table 2).

Table 2. Incidence of dysrhythmia in patient

\begin{tabular}{|l|l|l|l|l|}
\hline Variables & AF incidence & \multirow{2}{*}{ p-value (Chi-square) } \\
\cline { 3 - 4 } \multicolumn{2}{|c|}{} & Yes & No & \\
\hline \multirow{2}{*}{ Premature atrial contraction (PAC) } & Yes & 19 & 11 & \multirow{2}{*}{0.001} \\
\cline { 2 - 4 } & No & 66 & 165 & \\
\hline Premature ventricular contraction (PVC) & Yes & 35 & 45 & \multirow{2}{*}{0.010} \\
\cline { 2 - 4 } & No & 50 & 131 & \\
\hline Supraventricular tachycardia (PSVT) & Yes & 23 & 54 & \multirow{2}{*}{0.548} \\
\cline { 2 - 4 } & No & 62 & 122 & \\
\hline
\end{tabular}

\section{Discussion}

Postoperative atrial fibrillation (POAF) is common after cardiac surgery (30). The incidence of POAF varies from $10-50 \%$, and in our study, incidence of AF was observed in 32.6\% of the patients in ICU. Although it is not a lifethreatening rhythm disturbance and may present as self-limiting onset, it has major medical and economical implications (22). The pathogenesis of AF after cardiac surgery is believed to be multifactorial, including clinical variables and technical intraoperative factors $(30,31)$. The present study was aimed to determine the relationship between incidence of $\mathrm{AF}$ and duration of $\mathrm{CPB}$ pump in patients after coronary artery bypass graft surgery. According to studies, increasing CPB time can increase the complications. Confirming this issue, Cho (2017) with multivariate analysis, showed that longer CPB time ( $>60 \mathrm{~min}$, odds ratio: 7.47$)$ was a risk factor for lower radial pressure (32) and in the study of Radmehr (2010), in the group that had increase in preoperative creatinine, the CPB time was longer (33). The present study was a propos of the first objective of the research, which was "to determine the relationship between duration of the CPB pump ( $\mathrm{min}$ ) and incidence of AF after open heart surgery", the obtained results indicated no significant relationship between duration of the CPB pump and incidence of AF. This finding is consistent with the study of Thoren, which did not have a significant relationship CPB time with incidence of AF after heart surgery (34). Whereas, this result was not consistent with results of Helgadottir, or Mariscalco (25, 35); since, in these two studies, duration of CPB pump had significant relationship with incidence of AF after heart surgery. Difference in significance of the above-mentioned results might be due to the larger sample size in these studies or can be attributed to the fact that patients with various cardiac surgeries have been included in these evaluations. A propos of the second objective of the research, which was "to determine the relationship between incidence of AF and demographic characteristics", the statistical tests indicated significant relationship for variables of age and BMI; moreover, similar results were obtained by Prakasa Rao by investigating the effect of preoperative metoprolol on early initiation of AF, and Pilatis (2013), by predicting the AF in patients undergoing coronary arteries bypass surgery $(18,22)$. Furthermore, in a study by Van Oosten (2014), the variable of BMI was significant (36), but results of the statistical tests indicated no significant relationship between gender and incidence of AF $(\mathrm{p}>0.05)$, which was consistent with results of Gol Mohammadi (17). As for the other objective of the research, which was "to determine the relationship between AF incidence and duration of hospitalization (in days) in ICU after open heart surgery", the obtained results indicated a significant relationship. Accordingly, the studies conducted by Prakasa Rao (2016), Helgadottir (2012), Mariscalco (2014), and Sarzaeim (2014) showed that the number of days of hospitalization in ICU after open heart surgery has been significantly increased after incidence of $\mathrm{AF}(18,25,29,35)$. The increase in hospitalization days after the incidence of $\mathrm{AF}$ is due to complications of $\mathrm{AF}$ and hemodynamic instability, which leads to the continuation of treatment and hospitalization. In our study, the statistical tests indicated significant relationship between PVC-PAC and incidence of AF after open heart surgery $(\mathrm{p}<0.05)$; moreover, the study conducted by Sarzaeim to investigate the effect of vitamin $\mathrm{C}$ on prevention of AF, 
confirms the findings of the present study (29). Because the causes of AF incidence such as electrolyte disturbances and mechanical manipulation during surgery can cause other arrhythmias, in our research, the relation between clinical results and incidence of POAF (creatinine, sodium, potassium) indicated that only creatinine had a significant relationship, which was consistent with results of Melduni et al. who reported significant relationship between creatinine and incidence of AF. Moreover, in a study conducted to investigate the relationship between the increased mean serum creatinine level before and after surgery, Radmehr expressed that the increase in the mean level of serum creatinine before the operation, affected the increase in premature death and postoperative complications (29). In the present research, the results obtained for ejection fraction and duration of cross clamp and intubation indicated no statistically significant relationship, which was consistent with results of Thoren, Van Oosten, and Guenancia $(21,34,36)$. Furthermore, Ozcan's study showed no significant relationship between ejection fraction and postoperative AF (37). Lastly, it is necessary to mention regarding the retrospective of the study that all the factors are not under the control of the researcher, and there was no oversight on the completion of the records to record the required research data. The incompleteness of the data (Missing Data) recorded is one of the constraints. Also, some patients' records were out of the center for various reasons and were not available at the time of the implementation of the study. Undoubtedly, one of the most important limitations of retrospective studies is the lack of control of subversion.

\section{Conclusions}

Regarding the volume of the available information, the obtained results indicated no significant relationship between duration of $\mathrm{CPB}$ pump and incidence of $\mathrm{AF}$; however, by investigating and controlling other factors affecting incidence of AF, it would be possible to intervene in recognizing, preventing, and treating this type of common arrhythmia. Evidently, further studies in this field would lead to positive results in this regard.

\section{Acknowledgments:}

This study was supported by a grant from the Vice Chancellor for Research of the Hamedan University of Medical Sciences (project no.: 9406313516).

\section{Conflict of Interest:}

There is no conflict of interest to be declared.

\section{Authors' contributions:}

All authors contributed to this project and article equally. All authors read and approved the final manuscript.

\section{References:}

1) Dickson VV, Nocella J, Yoon HW, Hammer M, Melkus GD, Chyun D. Cardiovascular disease self-care interventions. Nursing research and practice. 2013; 2013: 407608. doi: 10.1155/2013/407608.

2) Riddell MA, Dunbar JA, Absetz P, Wolfe R, Li H, Brand M, et al. Cardiovascular risk outcome and program evaluation of a cluster randomised controlled trial of a community-based, lay peer led program for people with diabetes. BMC public health. 2016; 16(1): 864. doi: 10.1186/s12889-016-3538-3. PMID: 27558630 , PMCID: PMC4995663.

3) Gonzalez-Chica DA, Mnisi Z, Avery J, Duszynski K, Doust J, Tideman P, et al. Effect of Health Literacy on Quality of Life amongst Patients with Ischaemic Heart Disease in Australian General Practice. PloS one. 2016; 11(3): e0151079. doi: 10.1371/journal.pone.0151079. PMID: 26943925, PMCID: PMC4778924.

4) Bagheri K, Motamedi O, Aghadavoudi O, Akbari M. The Effects of Mean Arterial Pressure during Cardiopulmonary Bypass on Clinical and Paraclinical Parameters during and after Coronary Artery Bypass Graft Surgery. Journal of Isfahan Medical School. 2012; 29(169): 2574-83.

5) Baba-tabar H, Ebadi A, Karimi AA, Mokhtari-Nouri J, Tadrisi D, y M. Relationship complications after coronary artery bypass surgery with duration of intubation. Journal of Critical Care Nursing. 1388; 2(1): 31-3.

6) Bolourian AA, Beheshti-Monfared M, Gachkar L, Ghomeisi M, Shahzamani M, Foroughi M, et al. The preventive effects ofposterior pericardiotomy on atrial fibrillation after elective coronary artery bypass grafting. Tehran Univ Med J. 2011; 69(1): 29-35.

7) Sadeghi Meibodi A. Investigation the Outcomes of CABG in Patients with Left Ventricular Dysfunction. Journal of Guilan University of Medical Sciences. 2010; 19(73): 97-102. 
8) Wang S, Lv S, Guan Y, Gao G, Li J, Hei F, et al. Cardiopulmonary bypass techniques and clinical outcomes in Beijing Fuwai Hospital: a brief clinical review. ASAIO journal. 2011; 57(5): 414-20. doi: 10.1097/MAT.0b013e318227fa72. PMID: 21734556.

9) Cossu A, Suelzu S, Piu P, Orecchioni M, Bazzu G, Padua G, et al. Do on-and off-pump coronary bypass surgery differently affect perioperative peripheral tissue metabolism? Minerva anestesiologica. 2012; 78(1): 26-33. PMID: 21971436.

10) Luckraz H, Giri R, Wrigley B, Hennessy AM, Nicholas J, Nevill A. The use of the RenalGuard system in cardiac surgery with cardiopulmonary bypass: a first in man prospective, observational, feasibility pilot study. Open Heart. 2017; 4(2): e000669. doi: 10.1136/openhrt-2017-000669. PMID: 29071091, PMCID: PMC5640132.

11) Ellenberger C, Sologashvili T, Cikirikcioglu M, Verdon G, Diaper J, Cassina T, et al. Risk factors of postcardiotomy ventricular dysfunction in moderate-to-high risk patients undergoing open-heart surgery. Annals of cardiac anaesthesia. 2017; 20(3): 287-96. doi: 10.4103/aca.ACA_60_17. PMID: 28701592, PMCID: PMC5535568.

12) Youn Y, Kim J, Lee J, Choi C, Chang Y, Kwak H. The effect of intraoperative dexmedetomidine on acute kidney injury after pediatric congenital heart surgery. Medicine. 2017; 96(28): e7480. doi: 10.1097/MD.0000000000007480. PMID: 28700489, PMCID: PMC5515761.

13) Lemma MG, Coscioni E, Tritto FP, Centofanti P, Fondacone C, Salica A, et al. On-pump versus off-pump coronary artery bypass surgery in high-risk patients: operative results of a prospective randomized trial (onoff study). The Journal of thoracic and cardiovascular surgery. 2012; 143(3): 625-31. doi: 10.1016/j.jtcvs.2011.11.011. PMID: 22154798.

14) Zakkar M, Ascione R, James AF, Angelini GD, Suleiman MS. Inflammation, oxidative stress and postoperative atrial fibrillation in cardiac surgery. Pharmacology \& therapeutics. 2015; 154: 13-20. doi: 10.1016/j.pharmthera.2015.06.009. PMID: 26116810.

15) Sedighinejad A, Imantalab V, Mirmansouri A, Naderi Nabi B, Tarbiat M, Sadeghi AM, et al. Comparing the Effects of Isoflurane-Sufentanil Anesthesia and Propofol-Sufentanil Anesthesia on Serum Cortisol Levels in Open Heart Surgery with Cardiopulmonary Bypass. Anesthesiology and pain medicine. 2016; 6(6): e42066. doi: 10.5812/aapm.42066. PMID: 28975079, PMCID: PMC5560634.

16) Maesen B, Nijs J, Maessen J, Allessie M, Schotten U. Post-operative atrial fibrillation: a maze of mechanisms. Europace. 2012; 14(2): 159-74. doi: 10.1093/europace/eur208. PMID: 21821851, PMCID: PMC3262403.

17) Golmohammadi M, Esmaeeli Javid G, Farajzadeh H. Incidence and Risk Factors for Atrial Fibrillation after First Coronary Artery Bypass Grafting in Urumiyeh Imam Khomeini Hospital from 2006 to 2008. Iranian Cardiovascular Research Journal. 2010; 4(2): 86-90.

18) Prakasarao S, Ramani S, Manem N. Impact of preoperative metorrolol on the occurrence of new-onset atrial fibrillation after coronary artery bypass grafting. J Evid Based Med Health. 2016; 3(70): 3830-35. doi: $10.18410 /$ jebmh/2016/819.

19) Almassi GH, Pecsi SA, Collins JF, Shroyer AL, Zenati MA, Grover FL. Predictors and impact of postoperative atrial fibrillation on patients' outcomes: a report from the Randomized On Versus Off Bypass trial. The Journal of thoracic and cardiovascular surgery. 2012; 143(1): 93-102. doi: 10.1016/j.jtcvs.2011.10.003. PMID: 22054659.

20) Al-Shawabkeh Z, Al-Nawaesah K, Anzeh RA, Al-Odwan H, Al-Rawashdeh WA, Altaani H. Use of shortterm steroids in the prophylaxis of atrial fibrillation after cardiac surgery. Journal of the Saudi Heart Association. 2017; 29(1): 23-9. doi: 10.1016/j.jsha.2016.03.005. PMID: 28127215, PMCID: PMC5247295.

21) Guenancia C, Pujos C, Debomy F, Malapert G, Laurent G, Bouchot O. Incidence and Predictors of NewOnset Silent Atrial Fibrillation after Coronary Artery Bypass Graft Surgery. BioMed research international. 2015; 2015: 703685. doi: 10.1155/2015/703685. PMID: 26290873, PMCID: PMC4531157.

22) Pilatis ND, Anyfantakis ZA, Spiliopoulos K, Degiannis D, Chaidaroglou A, Vergou G, et al. The Role of BNP and CRP in Predicting the Development of Atrial Fibrillation in Patients Undergoing Isolated Coronary Artery Bypass Surgery. ISRN cardiology. 2013; 2013: 235018. doi: 10.1155/2013/235018. PMID: 24455301, PMCID: PMC3886441.

23) Mostafa A, El-Haddad MA, Shenoy M, Tuliani T. Atrial fibrillation post cardiac bypass surgery. Avicenna journal of medicine. 2012; 2(3): 65-70. doi: 10.4103/2231-0770.102280. PMID: 23826549, PMCID: PMC3697424.

24) Bockeria OL, Shvartz VA, Akhobekov AA, Kiselev AR, Prokhorov MD, Golukhova EZ, et al. Statin therapy in the primary prevention of early atrial fibrillation after coronary artery bypass grafting. Indian 
heart journal. 2016; 68(6): 792-7. doi: 10.1016/j.ihj.2016.04.002. PMID: 27931549, PMCID: PMC5143807.

25) Helgadottir S, Sigurdsson MI, Ingvarsdottir IL, Arnar DO, Gudbjartsson T. Atrial fibrillation following cardiac surgery: risk analysis and long-term survival. Journal of Cardiothoracic Surgery. 2012; 7(87): 1-7. doi: 10.1186/1749-8090-7-87.

26) Canale L, Clinic C, Mick S, Clinic C, Nair R, Mihaljevic T, et al. Atrial Fibrillation After Robotic Cardiac Surgery journal of Atrial Fibrillation. 2014; 7(1): 99-101.

27) Attaran S, Saleh HZ, Shaw M, Bond L, Pullan MD, Fabri BM. Comparing the outcome of on-pump versus off-pump coronary artery bypass grafting in patients with preoperative atrial fibrillation. Interactive cardiovascular and thoracic surgery. 2011; 13(3): 288-92. doi: 10.1510/icvts.2011.270249. PMID: 21700596.

28) Bessissow A, Khan J, Devereaux PJ, Alvarez-Garcia J, Alonso-Coello P. Postoperative atrial fibrillation in non-cardiac and cardiac surgery: an overview. Journal of thrombosis and haemostasis. 2015; 13 Suppl 1: S304-12. PMID: 26149040.

29) Sarzaeem M, Shayan N. Vitamin C in prevention of atrial fibrillation after coronary artery bypass graft: double blind randomized clinical trial. Tehran University Medical Journal. 2014; 71(12): 787-93.

30) Mihos CG, Santana O, Lamas GA, Lamelas J. Incidence of postoperative atrial fibrillation in patients undergoing minimally invasive versus median sternotomy valve surgery. The Journal of thoracic and cardiovascular surgery. 2013; 146(6): 1436-41. doi: 10.1016/j.jtcvs.2012.09.009. PMID: 23040194.

31) Patti G, Bennett R, Seshasai SR, Cannon CP, Cavallari I, Chello M, et al. Statin pretreatment and risk of inhospital atrial fibrillation among patients undergoing cardiac surgery: a collaborative meta-analysis of 11 randomized controlled trials. Europace. 2015; 17(6): 855-63. doi: 10.1093/europace/euv001. PMID: 25733550 .

32) Cho HJ, Lee SH, Jeong IS, Yoon NS, Ma JS, Ahn BH. Differences in perioperative femoral and radial arterial blood pressure in neonates and infants undergoing cardiac surgery requiring cardiopulmonary bypass. Jornal de pediatria. 2017.

33) Radmehr H1, Forouzannia SK, Bakhshandeh AR, Sanatkar M. Relation between Preoperative Mild Increased in Serum Creatinine Level and Early Outcomes after Coronary Artery Bypass Grafting. Acta Medica Iranica. 2011; 49(2): 89-94. PMID: 21598216.

34) Thoren E, Hellgren L, Stahle E. High incidence of atrial fibrillation after coronary surgery. Interactive cardiovascular and thoracic surgery. 2016; 22(2): 176-80. doi: 10.1093/icvts/ivv326. PMID: 26598007.

35) Mariscalco G, Biancari F, Zanobini M, Cottini M, Piffaretti G, Saccocci M, et al. Bedside Tool for Predicting the Risk of Postoperative Atrial Fibrillation After Cardiac Surgery: The POAF Score. Journal of the American Heart Association. 2014; 3(2): 1-9. doi: 10.1161/JAHA.113.000752. PMID: 24663335, PMCID: PMC4187480.

36) van Oosten EM, Hamilton A, Petsikas D, Payne D, Redfearn DP, Zhang S, et al. Effect of preoperative obstructive sleep apnea on the frequency of atrial fibrillation after coronary artery bypass grafting. American Journal of Cardiology. 2014; 113(6): 919-23. doi: 10.1016/j.amjcard.2013.11.047. PMID: 24462068.

37) Ozcan S. Relationship between atrial fibrilation and coronary bypass surgery. Pak Journal Medition Science. 2014; 30(3): 630-3. 